\title{
Quantification of Dose Nonuniformities by Voxel-Based Dosimetry in Patients Receiving ${ }^{90} \mathrm{Y}$-Ibritumomab-Tiuxetan
}

\author{
Francesco Cicone, Marco D’Arienzo, ${ }^{2,7}$ Andrea Carpaneto, ${ }^{3}$ Eleonora Russo, ${ }^{4}$ Angela Coniglio, ${ }^{5}$ \\ Angelika Bischof Delaloye, ${ }^{6}$ and Francesco Scopinaro ${ }^{1}$
}

\begin{abstract}
Objective: To assess the impact of nonuniform dose distribution within lesions and tumor-involved organs of patients receiving Zevalin ${ }^{\circledR}$, and to discuss possible implications of equivalent uniform biological effective doses (EU-BED) on treatment efficacy and toxicity. MATLAB ${ }^{\mathrm{TM}}$-based software for voxel-based dosimetry was adopted for this purpose.

Methods: Eleven lesions from seven patients with either indolent or aggressive non-Hodgkin lymphoma were analyzed, along with four organs with disease. Absorbed doses were estimated by a direct integration of singlevoxel kinetic data from serial tomographic images. After proper corrections, differential BED distributions and surviving cell fractions were estimated, allowing for the calculation of EU-BED. To quantify dose uniformity in each target area, a heterogeneity index was defined.

Results: Average doses were below those prescribed by conventional radiotherapy to eradicate lymphoma lesions. Dose heterogeneity and effect on tumor control varied among lesions, with no apparent relation to tumor mass. Although radiation doses to involved organs were safe, unexpected liver toxicity occurred in one patient who presented with a pattern of diffuse infiltration.

Conclusion: Voxel-based dosimetry and radiobiologic modeling can be successfully applied to lesions and tumor-involved organs, representing a methodological advance over estimation of mean absorbed doses. However, effects on tumor control and organ toxicity still cannot be easily predicted.
\end{abstract}

Key words: BED, EUD, ibritumomab-tiuxetan, lymphomas, NHL, radioimmunotherapy, voxel-based dosimetry, zevalin

\section{Introduction}

Tnternal dosimetry is constantly evolving with the use of radioimmunotherapy (RIT) for hematological malignancies. $^{1,2}$ Different dosimetric protocols have been developed for the two commercially available antilymphoma agents, ${ }^{131}$ I-tositumomab (Bexxar ${ }^{\circledR}$ ) and ${ }^{90} \mathrm{Y}$-ibritumomab-tiuxetan $\left(\right.$ Zevalin $\left.^{\circledR}\right)$. Because of the unpredictable biokinetics of ${ }^{131} \mathrm{I}$ - tositumomab, a pretherapy dosimetric study is mandatory in RIT with Bexxar (RIT-B) for tailoring the administered activity to deliver a total body dose of 75 or $65 \mathrm{cGy}$, according to the platelet count. ${ }^{3}$ In contrast, with the radiometal conjugate Zevalin (RIT-Z), the activity to be administered is based on patient weight and platelet counts rather than on pretherapeutic dosimetry. Indeed, it has been shown that, when performed at standard nonmyeloablative activities, only

\footnotetext{
${ }^{1}$ Nuclear Medicine, Department of Translational Medicine, Faculty of Medicine and Psychology, Sant'Andrea Hospital, "Sapienza" University of Rome, Rome, Italy.

${ }^{2}$ National Institute of Ionizing Radiation Metrology, ENEA, Centro Ricerche Casaccia, Rome, Italy.

${ }^{3}$ Hematology, Sant'Anna Hospital, Ronciglione (VT), Italy.

${ }^{4}$ Hematology, Department of Cellular Biotechnology and Hematology, Faculty of Medicine and Pharmacy, "Sapienza" University of Rome, Rome, Italy.

${ }^{5}$ Medical Physics, San Giovanni Calibita Hospital-Fatebenefratelli, Isola Tiberina, Rome, Italy.

${ }^{6}$ Nuclear Medicine, Centre Hospitalier Universitaire Vaudois and University of Lausanne, Lausanne, Switzerland.

${ }^{7}$ Department of Human Anatomy, Histology, Forensic Medicine and Orthopedics, "Sapienza" University of Rome.
}

Address correspondence to: Francesco Cicone; Nuclear Medicine, Department of Translational Medicine, Faculty of Medicine and Psychology, Sant'Andrea Hospital, "Sapienza" University of Rome; Via di Grottarossa 1035-1039, 00189 Rome, Italy E-mail: f.cicone@iol.it 
exceptionally $(<1 \%)$ would administration of RIT-Z be contraindicated because of excessive absorbed doses to organs at risk (OARs). ${ }^{4,5}$ Therefore, for standard treatments, many countries do not require a pretherapeutic dosimetry study.

In experimental clinical studies, RIT-Z has been also tested at higher, myeloablative activities, always showing a satisfactory toxicity profile. ${ }^{6.7}$ Despite the generally safe profile, in such trials, a pretherapy dosimetric study is strongly recommended because of possible, non-negligible organ toxicities. $^{8}$

Most dosimetric studies aim therefore at assessing the safety of RIT, whereas calculation of dose delivered to lesions has been rare thus far. A few reports on planar twodimensional (2D)-conjugated view-based dosimetry of patients receiving RIT-B failed to show a satisfactory prediction of tumor response. ${ }^{9,10}$ When a hybrid method was implemented combining planar imaging with single photon emission computed tomography (SPECT) and computed tomography (CT), a closer correlation between dose calculation and tumor volume reduction was achieved, especially for small lesions. ${ }^{9,10}$ These encouraging results were obtained in previously untreated tumors, in which it might be easier to find a dose-response relationship. Unfortunately, in patients with relapsed low-grade or transformed lymphomas, the efforts to establish a tumor dose-response relationship with a hybrid three-dimensional (3D) method were unsuccessful; nevertheless, with the voxel-based analysis, a trend toward better response with increasing uniformity of dose distribution was observed. ${ }^{11,12}$ Nonuniformities of dose distribution are indeed inherently related to the way of delivering radiation in RIT. If compared to external beam radiotherapy (EBRT), nonuniformities in RIT are less predictable and, together with the relatively low administered activity, may lead to a significant reduction of efficacy. This is especially the case for RIT-Z, where the administered activity is not based on dose-limiting toxicity.

One approach to addressing the effect of the heterogeneity of dose within the target volume is the use of the equivalent uniform biological effective dose (EU-BED or EUD for short), defined as the biologically effective dose, which, if delivered uniformly over the target volume, would yield the same number of surviving clonogens as the actual, nonuniform dose distribution. ${ }^{13}$

Some studies pioneered the systematic application of EUD in internal dosimetry and indicated the superiority of 3D biological approaches by demonstrating improved doseresponse correlations in RIT-B. ${ }^{14,15}$

Besides overcoming some drawbacks of 2D dosimetry, such as possible overlap between organs and poor differentiation of individual lesions with different biological features within the same bulk, one of the main advantages of 3D dosimetry is the possibility to evaluate the dose volume histograms (DVHs) which, in turn, allow for radiobiological modeling and for the evaluation of clinically relevant radiobiological parameters (i.e., the EUD).

To our knowledge, voxel-based dosimetry has not been applied yet to non-Hodgkin lymphoma (NHL) lesions receiving RIT-Z. Tumor 2D-estimated absorbed dose observed in clinical trials were in a wide range $(5.8-67.1 \mathrm{~Gy})^{16}$ and no correlation was found with tumor response, even with lesions not visualized on ${ }^{111}$ In-ibritumomab tiuxetan scans showing good sensitivity to treatment. ${ }^{17,18} \mathrm{~A}$ significant positive correlation between whole-body (WB) dose and progression-free survival was observed in a subgroup of patients treated with RIT-Z after the achievement of a response (nonmeasurable or low residual tumor burden) to first-line chemotherapy. ${ }^{19}$

The present study was carried out to explore the feasibility of dose distribution analysis within lesions and tumorinvolved organs of patients referred to RIT-Z because of relapsed/refractory NHL. MATLAB ${ }^{\mathrm{TM}}$-based software for voxel-based dosimetry was adopted for this purpose. ${ }^{20}$ Single-tumor responses as well as organ toxicity are discussed in view of the dosimetric results.

\section{Methods}

\section{Patient enrollment and planar imaging protocol}

Seven patients, referred to RIT-Z at the Nuclear Medicine Department of Sant'Andrea Hospital of Rome between February 2008 and April 2011 because of recurrent/ refractory NHL, were enrolled in a 3D dosimetry study in addition to a full planar dosimetry.

The planar dosimetric protocol consisted of several WB time point scans (10 minutes, 1, 24, 48, 72, 96, and 144 hours) acquired after the intravenous injection of ${ }^{111} \mathrm{In}$-Zevalin, $185 \mathrm{MBq}(5 \mathrm{mCi})$. Before tracer administration, all patients had received rituximab (Mabthera ${ }^{\circledR}$ ) $250 \mathrm{mg} / \mathrm{m}^{2}$ followed by a transmission scan using an ${ }^{111}$ In flood source for attenuation correction. WB images were acquired on a dual-head 5/ 8 "-thick crystal MEGP-collimated Forte camera (Philips) with a matrix size of $512 \times 512$, scan speed $10 \mathrm{~cm} / \mathrm{min}$.

To be included in the additional SPECT protocol, patients were required to show pathological tumor sites at pretherapy positron emission tomography (PET/CT), acquired within 1 month before RIT. After ethical approval, all patients gave separate informed consent for the planar dosimetry as well as for the additional SPECT-based study.

Patients' characteristics and tumor sites are summarized in Table 1 . The median age was 58 years (range: $32-67$ years). Patients A and B had an untreated relapse of low-grade (grade 2) follicular lymphoma (FL), while patient $C$ had a grade $2 \mathrm{FL}$, which was refractory to the last previous treatment. Four patients had aggressive NHL: patients D, F, and $\mathrm{G}$ had a refractory diffuse large B-cell lymphoma, while patient $\mathrm{E}$ had transformed grade $3 \mathrm{~b}$ FL. The median number of prior lines of chemoimmunotherapies was 3 (range: 2-11), with a median of two prior rituximab-containing regimens (range: 1-4). Previously, in their treatment course, patients D and $\mathrm{E}$ had received involved-field EBRT and myeloablative chemotherapy followed by stem cells rescue. Patient $G$ had received autologous bone marrow transplantation as well.

\section{SPECT imaging protocol}

SPECT images (32 steps, 30 seconds/step) encompassing target lesions were acquired 48, 72, 96, and 144 hours after the administration of the tracer. In some cases of both upper and subdiaphragmatic tumor involvement, not covered by only one SPECT field of view (e.g., patients D, F, and G), it was not possible to acquire serial images of all lesions because of patient discomfort. In such cases, SPECTs were acquired over the biggest or most visible lesions. A matrix size of $64 \times 64$, corresponding to a voxel dimension of 


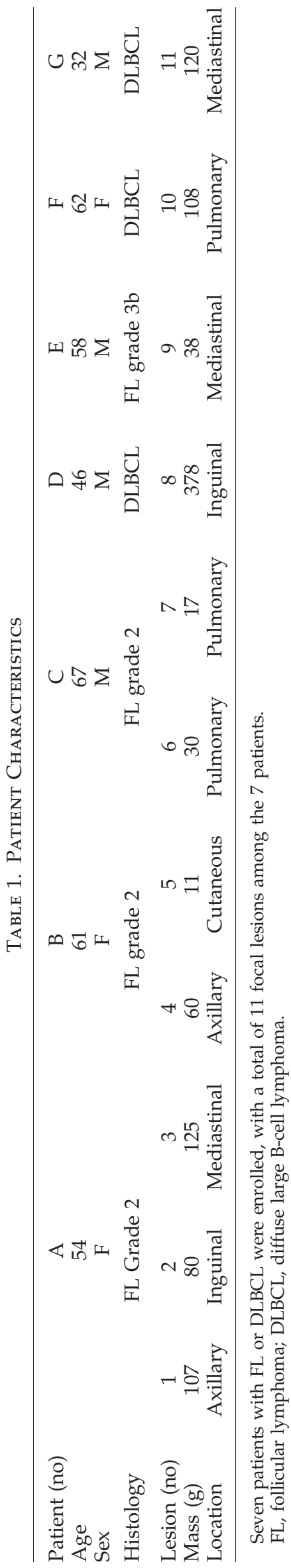

$9.3 \times 9.3 \times 9.3 \mathrm{~mm}^{3}, 0.8 \mathrm{~mL}$ volume), was used. SPECTs were reconstructed with OSEM algorithm (8 substeps, 12 iterations). ${ }^{20}$ With the aforementioned acquisition parameters, the spatial resolution of our SPECT scanner was determined to be $\sim 1.8 \mathrm{~cm}$.

The triple energy window (TEW) technique was used for scatter correction, as described elsewhere. ${ }^{20,21}$ TEW algorithm consists of two main $20 \%$ energy windows centered at the ${ }^{111}$ In photopeaks (171 and $245 \mathrm{keV}$ ) and two 6\% energy subwindows on both sides of the main window, centered at 155,190 , and $222 \mathrm{keV}$, respectively. With the main windows, the total counts $C_{\text {tot }}$ can be estimated, while the scattered components $\mathrm{C}_{\mathrm{sca}}$ for both photopeaks can be assessed through the following relations:

$C_{\text {scat_ } 171}=\left(\frac{\mathrm{C}_{155 \mathrm{keV}}+\mathrm{C}_{190 \mathrm{keV}}}{2}\right) \cdot \frac{w_{171}}{w_{155 / 190}} C_{\text {scat_245 }}=\left(\frac{\mathrm{C}_{222 \mathrm{keV}}}{2}\right) \cdot \frac{w_{245}}{w_{222}}$

where $C_{155 \mathrm{keV}}, \mathrm{C}_{190 \mathrm{keV}}, \mathrm{C}_{222 \mathrm{keV}}$ are the counts in the three subwindows, which are located, respectively, at the sides of the main windows and $w_{171}, w_{245}, w_{155 / 190}, w_{222}$ are the width of the main windows and the scatter windows, respectively. The count of primary photons is given by $C_{\text {prim }}=$ $C_{\text {tot }}-\left(C_{\text {scat_171 }}+C_{\text {scat_245 }}\right)$.

For each tumor, attenuation correction was applied by deriving an attenuation map from images obtained with the X-ray CT scan. A low-dose CT acquisition protocol for image attenuation correction was adopted with $120 \mathrm{kV}$ and $70 \mathrm{~mA}$. Images were reconstructed at $0.9-\mathrm{cm}$ section thickness with an iterative reconstruction algorithm and a $512 \times 512$ matrix; the matrix was then scaled to $64 \times 64$ to match SPECT slices. Linear attenuation coefficients for the two ${ }^{111}$ In peaks were determined converting Hunsfield units by using the bilinear relationships proposed by Brown et al. ${ }^{22}$ For each material, the effective attenuation coefficient was determined as a weighted sum of the 172 and $247 \mathrm{keV}$ attenuation coefficients using weights 0.49 and 0.51 , respectively.

The serial ${ }^{111}$ In images were aligned using a mutual information algorithm. ${ }^{20}$

After corrections, in sequence, for scatter first and for attenuation thereafter, correction for partial volume effect (PVE) was also performed according to a previously reported method. $^{20}$

Volume corrections were performed by applying a volumedependent intensity threshold on the outlined slices ${ }^{23-25}$ basing on individual tumor masses estimated by contouring CT axial slices with the Varian Eclipse ${ }^{\mathrm{TM}}$ Treatment Planning System for EBRT. Loss of activity was compensated through the evaluation of volume-dependent recovery coefficients (RCs). RCs were considered $<1$ for the lesions with target volumes larger than $50 \mathrm{~mL}$ (See Table 1). Similar corrections have been previously described ${ }^{26}$ and applied to patient studies. ${ }^{9,15}$

\section{Tumor 3D dosimetry}

Tumor doses were estimated by means of MATLAB ${ }^{\text {TM}}-$ based software, allowing for a direct integration of voxel-based kinetic data from SPECT. The original counts were translated into activity inside the voxels through a phantom-based procedure that allowed the counts-to-activity calibration factor (cps/MBq) to be determined. A comprehensive description of 
the phantom procedures can be found elsewhere. ${ }^{20}$ A homogeneous activity distribution was assumed inside each voxel.

To estimate the cumulated activity, individual tumors were contoured on registered axial SPECTs acquired at different time points by two experienced readers. We decided not to consider images before 48 hours, since no specific uptake was generally seen on tumor sites before that time point, and this might have affected proper tumor contouring. The same choice was adopted for tumor 2D dosimetry. Therefore, although estimated doses might be affected by a small error due to the lack of image sampling before 48 hours, SPECT and planar dose values are comparable.

The cumulated activity concentration in each voxel was computed for each time point by fitting activity with a monoexponential function, and then convolved with voxel S-values to provide 3D maps of the absorbed dose. The 3D dose distributions were then used to derive DVHs. 3D dose calculations were performed using the voxel $S$ value approach, ${ }^{27}$ where S-values for voxel dimension of $9.3 \times 9.3 \times 9.3 \mathrm{~mm}^{3}$ were calculated by means of the Monte Carlo N-particle radiation transport computer code (MCNP, version 4c). ${ }^{20}$ SPECT calibration procedures, description, and validation of correction techniques and results of S-value calculations have been extensively presented elsewhere. ${ }^{20}$

\section{Tumor response assessment}

Three months after RIT-Z, patients underwent either PET/ CT or contrast-enhanced CT (CECT) for response evaluation. While CECT was performed in different centers according to the referring hematological institution, pre- and post-therapy PET/CT were acquired on the same camera (Philips, Gemini). Acquisitions (speed: $2.5 \mathrm{~cm} / \mathrm{min}$, axial field of view: $18 \mathrm{~cm}$, $50 \%$ BED overlap) started 60 minutes after the i.v. injection of ${ }^{18}$ F-fluorodeoxyglucose (FDG), 3.7 MBq/Kg $( \pm 10 \%)$. Patients had been fasting for at least 6 hours and plasma glucose levels were checked to be $\leq 140 \mathrm{mg} / \mathrm{dL}$ before FDG administration.

Response criteria were defined according to the Imaging Subcommittee of International Harmonization Project in Lymphoma. ${ }^{28}$ The presence of residual masses $<$ or $\geq 2 \mathrm{~cm}$ in diameter was considered as a complete response (CR) if the corresponding FDG uptake was lower compared with the surrounding background or mediastinal blood pool uptake, respectively. Conversely, if significant glucose metabolism was present at the site of residual masses of any size, the definition of a partial response, stable disease, or progressive disease (PD) was based on CECT morphological findings. ${ }^{29}$ In such cases, maximum FDG Standardized Uptake Value (SUV max) measured before and after RIT-Z was reported.

\section{Tumor-involved organs: $3 D$ versus $2 D$ dosimetry}

In addition to nodal lesions, 3D dosimetry was also performed using the method described above on the liver and spleen of patients $F$ and $G$, to evaluate the dose nonuniformity within these organs. Both patients had stage 4 disease involving the liver and spleen, which therefore were likely to show areas of heterogeneous uptake. Although not clearly identifiable on the pretherapy dosimetric scan, organ involvement was clinically suspected and subsequently confirmed by PET/CT.

Voxel-based calculations were compared with standard 2D dosimetry performed by applying the conjugate-view technique over several time point planar scans according to MIRD pamphlet $16 .^{30}$ Scatter and background correction were performed according to the pseudoextrapolation number technique ${ }^{30}$ and Buijs' method, ${ }^{31}$ respectively, while attenuation correction was performed through WB transmission scans using an ${ }^{111}$ In flood source. Correction for PVE was not applied to planar dosimetry.

For each time point, tumor/organ uptake was expressed relative to the body counts obtained 10 minutes after the injection. Cumulated activities were calculated by fitting the image-derived time activity to a biesponential function and analytically integrating the resulting functions. To obtain the absorbed dose, cumulated activity of organs was then combined with the appropriate dose-conversion S-values retrieved from OLINDA software. ${ }^{32}$ Individual organ masses were determined by axial CT contouring and input into OLINDA. The planar dosimetry protocol adopted here has been previously described. ${ }^{20}$

The quality of monoexponential and biexponential fit was assessed by evaluating the coefficient of determination $r^{2}$ for each target region. The same approach has been previously used. ${ }^{20}$ Single and biexponential fitting techniques were in good agreement, being in all cases $>0.92$. Therefore, the dose error due to the use of different fitting functions was assumed to be negligible.

\section{Evaluation of nonuniform dose distributions}

As reported by $\mathrm{O}^{\prime}$ Donoghue, ${ }^{33}$ the EUD formalism may be used to incorporate the biologic effects. By introducing the radiobiologic parameters $\alpha$ and $\beta$, that is, the sensitivity per unit dose and per unit dose squared, respectively, at the voxel level, the EUD model converts the heterogeneous $D$ distribution into an uniform BED that would produce the same biologic effect as the nonuniform BED distribution. Therefore, this formalism provides a single value that fully describes the biologic effect of a nonuniform dose distribution and that can be used to compare different dose distributions. $^{33}$

For a given dose distribution, the equivalent uniform biologically effective dose can be calculated as follows:

$$
E U D=-\frac{1}{\alpha} \ln (S)=\frac{1}{\alpha} \ln \left[\int_{0}^{\infty} P(\psi) e^{-\alpha \psi} d \psi\right]
$$

where $\psi$ represents $\mathrm{BED}, P(\psi)$ the probability density function, $\mathrm{S}$ is the overall surviving fraction for the nonuniform distribution $P(\psi), e^{-\alpha \psi_{i}}$ is the surviving fraction produced by a given BED, and $\alpha$ is the radiosensitivity factor. For each voxel, the BED was calculated as $\psi_{i}=D_{i} \cdot R E_{i}$, where $D_{i}$ is the voxel dose and $R E_{i}$ the relative effectiveness evaluated as reported by Barone et al. ${ }^{34}$

The relative effectiveness RE depends upon the $\alpha / \beta$ ratio and the repair half time for sublethal damage $\left(\mathrm{T}_{\mu}\right)$. In the present work, the standard values $\alpha / \beta=10 \mathrm{~Gy}$ and $\mathrm{T}_{\mu}=0.5$ hours were considered for tumor dosimetry, while $\alpha / \beta=$ $3 \mathrm{~Gy}$ and $\mathrm{T}_{\mu}=3$ hours were used both for the liver and spleen. $^{35}$

The radiosensitivity $\alpha$ coefficient in Equation 1 was assumed to be $0.35 \mathrm{~Gy}^{-1} .33$ This value is not only limited to RIT treatments, since a similar $\alpha$ parameter has been previously 


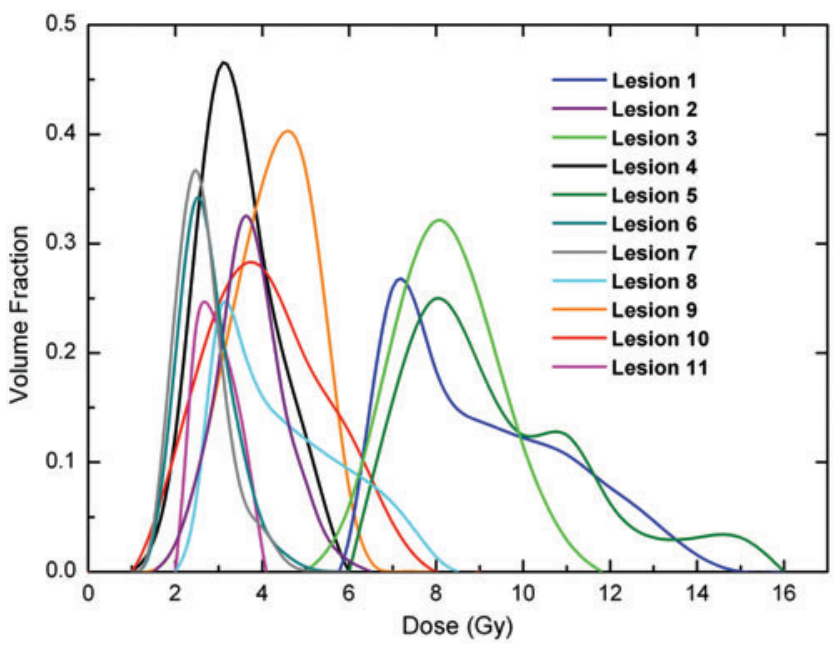

FIG. 1. Differential dose volume histograms (DVHs) for lesions, normalized so that the area under the curve is 1 . Dose distribution, reported as a differential DVH, provides an accurate assessment of homogeneity in the volume of interest. Each DVH represents the tumor volume receiving a dose given by the bin. Bin doses are shown on the horizontal axis (1 Gy-bin size), whereas structure volumes-reported as a fraction of the whole volume-are on the vertical axis. Color images available online at www.liebertpub.com/cbr

used for systemic radionuclide therapies with various $\beta$ emitters $^{36-39}$ and with radioiodine as well. ${ }^{12}$ By considering radiosensitivity $\alpha=0.35 \mathrm{~Gy}^{-1}$, Kalogianni and colleagues assessed the effect of nonuniform activity distributions at a multicellular scale in terms of DVHs, BED, and EUD for ${ }^{32} \mathrm{P}$, ${ }^{90} \mathrm{Y}$, and ${ }^{131} \mathrm{I} \mathrm{I} .{ }^{39}$ These authors showed that, for ${ }^{32} \mathrm{P}$ and ${ }^{90} \mathrm{Y}$, the loss of therapeutic effect (LTE) is less than for ${ }^{131} \mathrm{I}$. In fact, since ${ }^{32} \mathrm{P}$ and ${ }^{90} \mathrm{Y}$ have longer emission ranges, the increased effect of the cross-fire produces more uniform dose distributions in larger volumes. They also reported that ${ }^{90} \mathrm{Y}$ is likely to be less effective than ${ }^{32} \mathrm{P}$ and ${ }^{131} \mathrm{I}$, because of its shorter physical half-life resulting in lower mean BED values.

The probability density function $P(\psi)$ corresponds to the differential BED distribution, or differential BED volume histograms (BVHs), normalized so that the area under the curve is 1, and is retrieved by differential DVH (See Fig. 2). To quantify dose uniformity both in tumor regions and OARs, for each target area, a heterogeneity index (HI) was defined as the ratio of the dose delivered to $5 \%$ and $95 \%$ of the target volume. A larger HI indicates greater dose heterogeneity inside the lesion. ${ }^{40}$ For each lesion, the LTE for a given dose distribution was calculated as the difference between the mean BED and the EUD. ${ }^{33}$

\section{Results \\ Dosimetry: single tumors}

Differential DVHs, normalized so that the area under the curve is 1, are shown in Figure 1. BEDs of low-grade lesions were in the range 2.62-9.68 Gy (median: 3.75 Gy), while they were in the range 2.86-4.33 Gy (median: 4.18Gy) in highgrade lymphomas.

As expected, EUDs were systematically lower than BEDs, being in the range 2.57-9.04 Gy (median: 3.66 Gy) and 2.824.17 (median: $3.9 \mathrm{~Gy}$ ) for low- and high-grade lymphomas, respectively. The complete list of values is reported in Table 2 , together with HI, LTE, and surviving fractions $(S)$ following uniform irradiation of a cell population $S=e^{-\alpha E U D}$.

As a general rule, as the distribution becomes less uniform, the EUD decreases and-as reported by $\mathrm{O}^{\prime}$ Donoghue ${ }^{33}$ the higher the absorbed dose, the higher the effect of dose nonhomogeneity on therapeutic effect (see Table 2). Indeed, our data confirm that the absolute LTE is greater for higher BED values (Fig. 3). ${ }^{33}$

\section{Dosimetry: organs}

Table 3 shows 2D and 3D dose calculations for NHL involved organs of patients $\mathrm{F}$ and $\mathrm{G}$, while the corresponding DVHs are depicted in Figure 4. Since the concept of therapeutic effectiveness is related only to tumor, LTE and S for tumor-involved organs have not been reported in Table 3. Liver BED of patient $G$ was higher compared with patient $F$ (8.46 vs. $4.15 \mathrm{~Gy}$, respectively). Heterogeneity was higher $(\mathrm{HI}=1.9$ vs. 1.6$)$ in the liver of patient $\mathrm{F}$, showing multiple single metastases (See Fig. 5), than in the liver of patient G, whose liver involvement was diffuse.

Table 2. Results of Tumor Dosimetry

\begin{tabular}{rcccccccc}
\hline & & & & & & \multicolumn{2}{c}{ LTE } \\
\cline { 5 - 6 } Lesion & Mass $(g)$ & BED $(G y)$ & EUD $(G y)$ & $S$ & HI & (Gy) & (\%) & Outcome \\
\hline 1 & 107 & 9.26 & 8.63 & 0.05 & 1.91 & 0.63 & -6.80 & CR \\
2 & 80 & 3.75 & 3.66 & 0.28 & 3.58 & 0.09 & -2.40 & CR \\
3 & 125 & 8.39 & 8.03 & 0.06 & 1.77 & 0.36 & -4.29 & CR \\
4 & 60 & 3.44 & 3.31 & 0.31 & 4.43 & 0.13 & -3.78 & CR \\
5 & 11 & 9.68 & 9.04 & 0.04 & 1.97 & 0.64 & -6.61 & CR \\
6 & 30 & 2.75 & 2.69 & 0.39 & 3.57 & 0.06 & -2.18 & PD \\
7 & 17 & 2.62 & 2.57 & 0.41 & 3.39 & 0.05 & -1.91 & PD \\
8 & 378 & 4.20 & 3.96 & 0.25 & 3.07 & 0.24 & -5.71 & PD \\
9 & 38 & 4.33 & 4.17 & 0.23 & 4.13 & 0.16 & -3.70 & SD \\
10 & 108 & 4.16 & 3.85 & 0.26 & 5.91 & 0.31 & -7.45 & Unknown \\
11 & 120 & 2.86 & 2.82 & 0.37 & 1.55 & 0.04 & -1.40 & SD \\
\hline
\end{tabular}

$\mathrm{BED}$, biological effective doses; EUD, equivalent uniform biological effective dose; HI, heterogeneity index; LTE, loss of therapeutic effect; $\mathrm{CR}$, complete response; $\mathrm{PD}$, progressive disease; $\mathrm{SD}$, stable disease. 


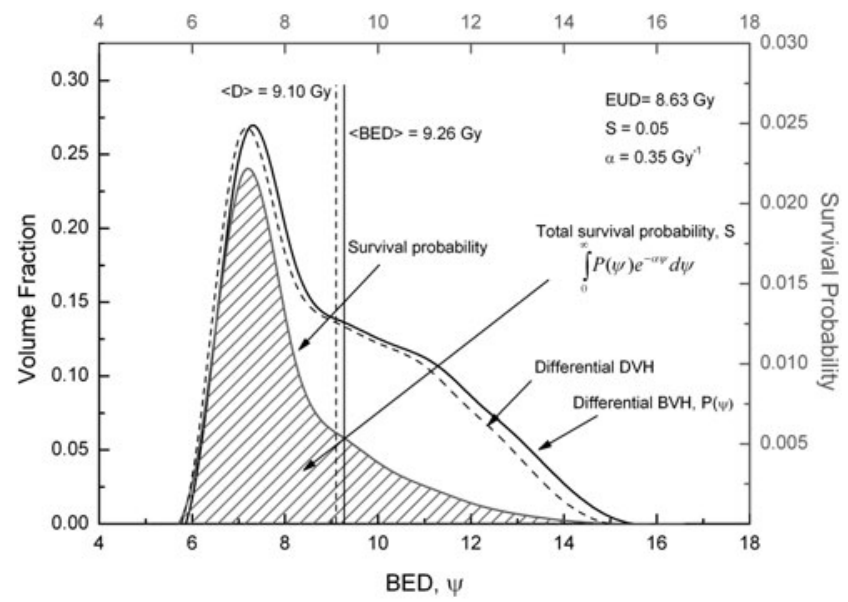

FIG. 2. Lesion 1: differential DVH (dotted line) and biological effective doses (BED) volume histogram $P(\psi)$ normalized such that the area under the curve is 1 (BED volume histogram [BVH], black solid line). Average absorbed dose and BED are also reported. The solid line outlining the diagonals represents the resulting distribution of survival probability calculated for tumor radiosensitivity parameter of $0.35 \mathrm{~Gy}^{-1}$. The smaller, dashed area represents the overall surviving fraction for the nonuniform distribution $P(\psi)$, calculated by the Laplace transform of the BVH (equation in the graph). EUD, equivalent uniform biological effective dose.

The two spleens received similar doses (4.14 vs. $4.66 \mathrm{~Gy}$ ). Again, the organ with more focal pattern of involvement (patient G, See Fig. 6) showed higher $\mathrm{HI}(\mathrm{HI}=1.42$ vs. 1.75) than the liver whose tumor involvement was fairly diffuse (patient F, see Fig. 5). In all the 4 organs analyzed, 2D dosimetry gave lower doses than 3D dosimetry (See Table 3).

\section{Response assessment}

Nine (number $1-7,9,11$ ) out of 11 lesions were classified by combining information from post-therapy PET/CT and CECT. Lesion 8 (patient D) was restaged on the basis of physical examination and CT only, before the patient was

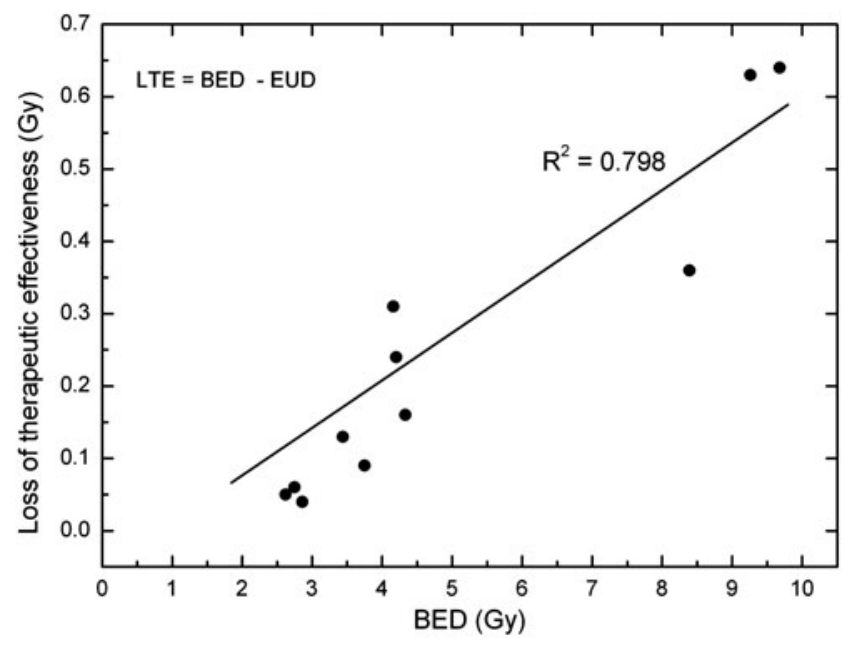

FIG. 3. Absolute loss of therapeutic effect (LTE) versus BED in lesions. LTE is worse for larger mean BED values.
Table 3. Dose Estimations for Liver and Spleen of Patients F AND G

\begin{tabular}{lccccccc}
\hline & & $\begin{array}{c}\text { Mass } \\
\text { Organs }\end{array}$ & $\begin{array}{c}\text { 2D dose } \\
(\mathrm{Gy})\end{array}$ & $\begin{array}{c}3 \mathrm{D} \text { dose } \\
(\mathrm{Gy})\end{array}$ & $\begin{array}{c}\text { BED } \\
(\mathrm{Gy})\end{array}$ & HI & $\begin{array}{c}\text { EUD } \\
(\mathrm{G} y)\end{array}$ \\
\hline \multirow{4}{*}{ Liver } & $\mathrm{F}$ & 2130 & 2.40 & 4.10 & 4.15 & 1.90 & 4.0 \\
& $\mathrm{G}$ & 2230 & 5.50 & 8.30 & 8.46 & 1.60 & 8.13 \\
Spleen & $\mathrm{F}$ & 1337 & 2.40 & 4.10 & 4.14 & 1.42 & 4.09 \\
& & 415 & 3.30 & 4.60 & 4.66 & 1.75 & 4.50 \\
\hline
\end{tabular}

referred to subsequent salvage therapy. Both patients $F$ and $\mathrm{G}$ died several months after RIT-Z. While the response of lesion 11 (patient $\mathrm{G}$ ) could be evaluated by post-therapy PET/CT (Fig. 6), the death of patient F occurred before response of lesion number 10 and post-therapy status of parenchymal disease could be classified.

Five low-grade lymphoma lesions (number 1-5) showed a CR; lesions number 6, 7, and 8 (patients $C$ and $D$ ) progressed after RIT-Z. Lesions number 9 and 11 (patients $E$ and G) were stable but, due to the appearance of newly involved sites, those patients were considered globally progressive. Preand post-therapy SUV max values of lesion number 9 were 11.1 and 5, respectively (SUV reduction $=55 \%$ ), while they were 25.7 and 3.8 in lesion number 11 (SUV reduction $=96 \%$ ) (Fig. 6). Lesion 10 could not be classified in terms of response because of the early death of patient F. Patient F died of a pulmonary edema during bone marrow aplasia, with febrile neutropenia and severe diarrhea; death occurred 2 months after RIT-Z and was attributed to hematological toxicity, no autopsy was performed. Patient G expired 3.5 months after RIT-Z. A direct cause of death was pulmonary edema; however, concomitant hepatic iatrogenic necrosis and NHL liver involvement were diagnosed at autopsy. The observed liver toxicity was not predictable based on the estimated absorbed dose (Table 3). Both the liver and spleen of patient $\mathrm{G}$ showed a dramatic volumetric increase from pre- to post-therapy PET/ CT (2230 vs. $3420 \mathrm{~g}$ for liver, $415 \mathrm{vs.} 1184 \mathrm{~g}$ for spleen); however, while on post-therapy PET/CT, the spleen was clearly abnormal, liver glucose uptake was more homogeneous than before, without signs of disease progression (Fig. 6).

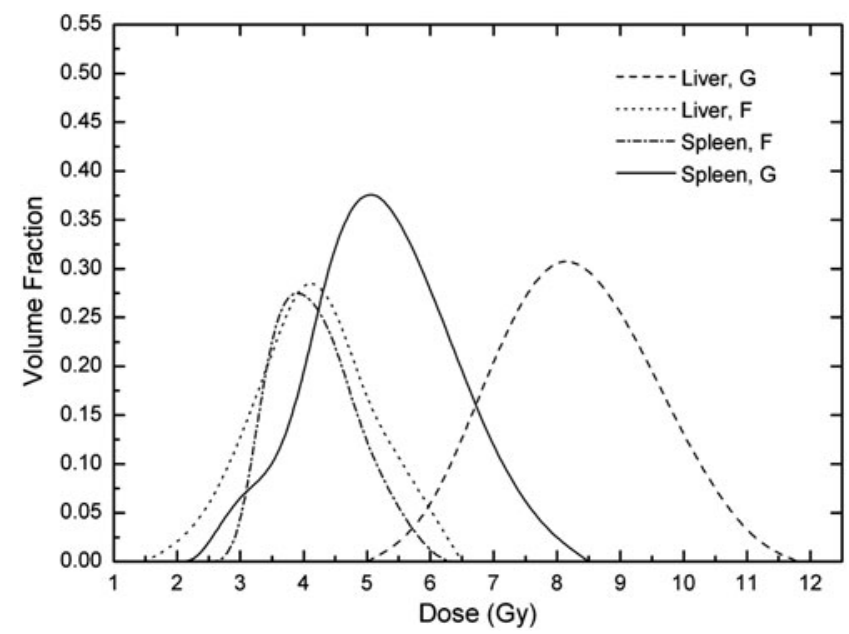

FIG. 4. Differential DVHs for organs at risk. 
FIG. 5. Pretherapy FDG PET/CT of patient F. A pattern of focal tumor involvement is shown, with multiple hepatic lesions (coronal and axial slices). Color images available online at www.liebertpub .com/cbr

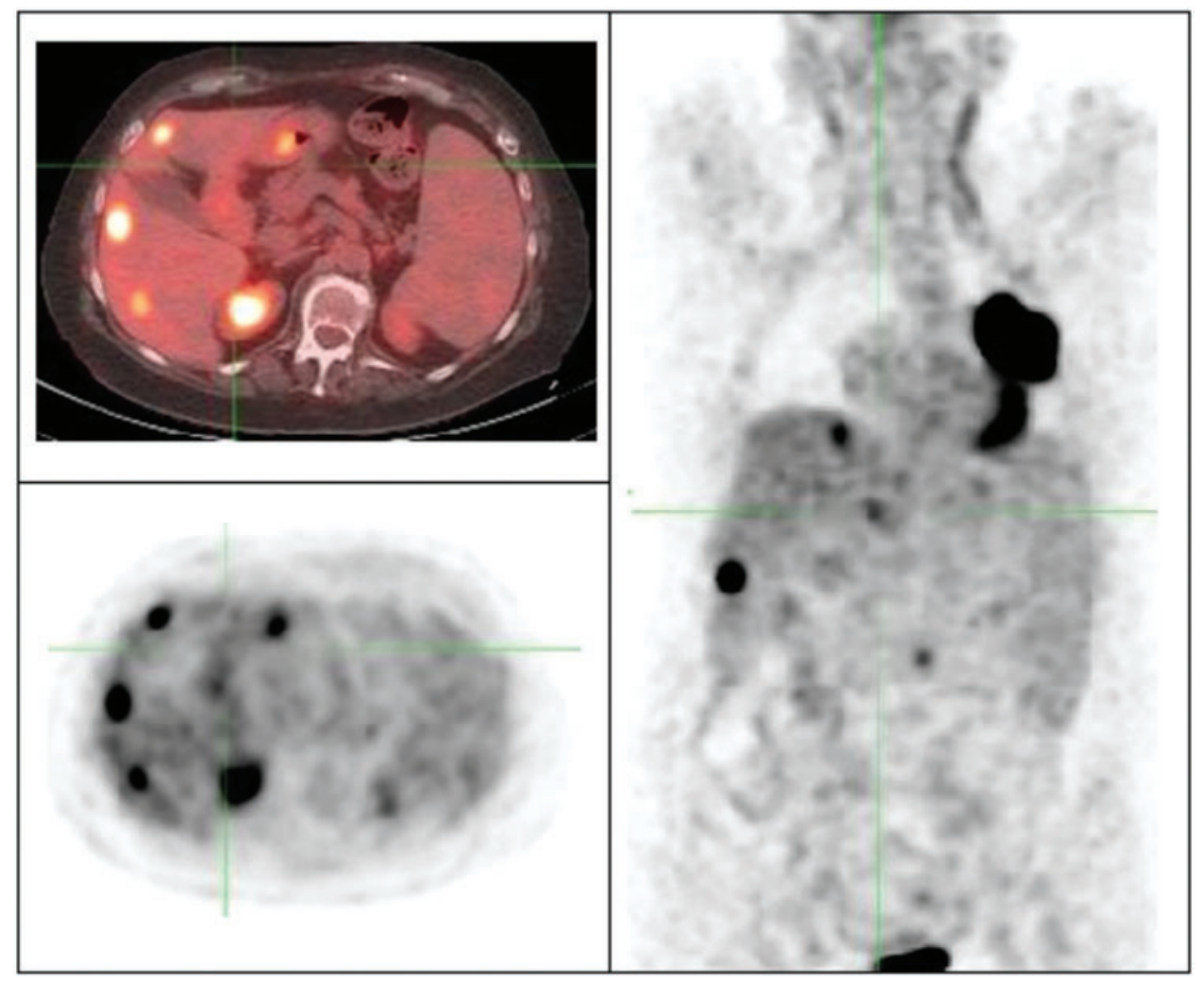

\section{Discussion}

To our knowledge, this is the first report analyzing dosevolume distributions and applying the EUD concept to NHL lesions receiving RIT-Z. ${ }^{41}$ We are aware that the population under study and the incomplete sampling of tumor lesions would not allow a robust dose-response analysis on patient basis, which therefore was not attempted. Rather, our aim was to show the feasibility and discuss some implications of such approach, as we believe that the application of radiobiologic modeling to patient-specific 3D imaging may provide new insights into tumor and organ dosimetry. ${ }^{42}$

Our results confirm that dose nonuniformities in RIT are quite large, resulting in higher $\mathrm{HI}$ values compared to
A

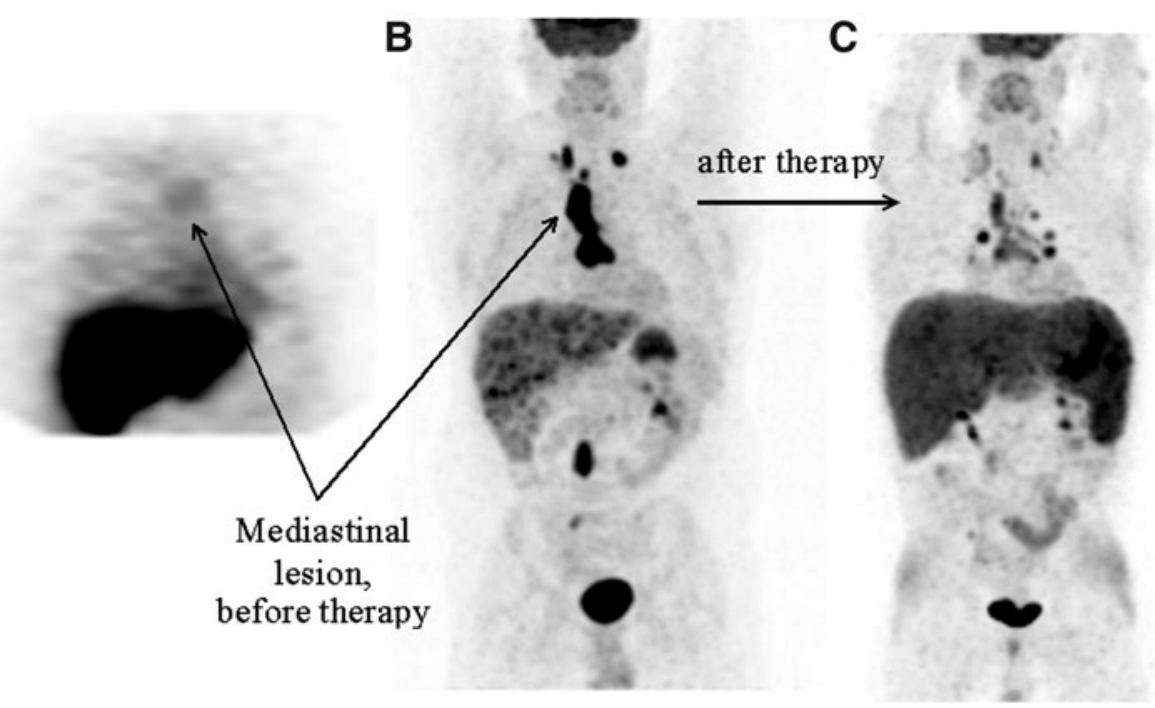

FIG. 6. Pre- and post-therapy imaging of patient G. (A) pretherapy ${ }^{111}$ In-single photon emission computed tomography $(\mathrm{SPECT}) ;(\mathbf{B}, \mathbf{C})$ pre- and post-therapy PET/CT. Although ${ }^{111} \mathrm{In}-\mathrm{SPECT}$ showed poor uptake (A, coronal slice) in the mediastinal lesion, maximum FDG Standardized Uptake Value (SUV max) decreased from 25.66 before (B) to 3.8 after therapy (C). $\mathrm{C}$ depicts the good response of abdominal as well as supraclavicular nodes, whereas hilar nodes progressed bilaterally. Diffuse hepatic involvement was present before therapy (B). After RIT-Z, while liver uptake was almost homogeneous, the spleen showed clear disease progression (C). 
conventional EBRT. Moreover, the intratumoral distribution of ${ }^{90}$ Y-ibritumomab-tiuxetan varies widely among tumors and is apparently not dependent on tumor mass. As shown by the comparisons between lesions 1 and 10 and between lesions 1 and 5, respectively, $\mathrm{HI}$ of lesions with similar mass can be very different, and $\mathrm{HI}$ of lesions with very different mass can be similar (See Table 2). As previously described, LTE is lower for lesions receiving low average doses. ${ }^{33}$ However, the contribution of predicted LTE and $S$ on tumor control will hopefully be clarified in larger series of patients. Interestingly, despite $28 \%$ and $31 \%$ estimated surviving tumor cells in lesions number 2 and 4, respectively, both responded completely to treatment, and remain in CR 10 months after RIT-Z.

Consistent with most previous reports in NHL, the response observed in single lesions was apparently not dependent on the calculated dose, and varied according to tumor aggressiveness. Of note, probably because of the small patient sample size, the tumor dose range observed was smaller than previously reported, ${ }^{16}$ and all lesions received doses that would have been considered suboptimal according to the current standard of EBRT (30-36 Gy). The need for 30-36 Gy has been questioned in case of both indolent and aggressive lymphomas, although the evidence supporting the efficacy of low radiation doses is stronger for indolent than for aggressive lymphomas. ${ }^{43,44}$ It is well known that the efficacy of RIT-Z is low in patients with aggressive lymphomas, especially those with previous exposure to rituximab. ${ }^{45}$ Unfortunately, in case of progression after optimized rituximab-including treatments, the efficacy of RIT- $Z$ is reduced in patients with indolent lymphomas as well. ${ }^{46,47}$ A possible acquired resistance to immunotherapy, together with the low radiation doses, might explain the treatment failure of patient $C$, whereas the tumors of patients A and B were more sensitive, as they had already achieved CRs after prior optimized treatments. A comprehensive understanding of all the variables affecting tumor sensitivity is a long way away; even more distant is a tailored treatment based on tumor dose predictions. However, optimization of doses in RIT would be welcome, so the technique developed here warrants further investigation.

A method for 3D dose calculation could be successfully applied to organs with disease involvement where a heterogeneous dose distribution is more likely than in healthy organs. Thus, we conducted a separate analysis on the liver and spleen of two patients ( $F$ and $G$ ) with extensive tumor involvement, comparing voxel-based 3D calculations with 2D dosimetry. Although most studies indicate that 3D doses are generally lower than those obtained with planar dosimetry, ${ }^{48}$ the opposite was found in our series. Although this observation might be serendipitous, we believe that it could be potentially related to the massive organ tumor involvement. Interestingly, when the same methodology and the same corrections were applied to organs without tumor involvement, 3D determined absorbed doses turned out to be generally lower than $2 \mathrm{D} .^{20}$

Both patients $F$ and G had PD after several courses of chemoimmunotherapy; patient $\mathrm{G}$ had already received high-dose chemotherapy with stem cell rescue and had prior history of drug abuse (cannabinoids and cocaine) since the age of 14 . Before RIT, liver function tests and viral serology (HBV, HCV, HIV) were unremarkable in both patients. The liver toxicity of patient $G$ was not curable and significantly worsened his prognosis. The reason for such toxicity was not entirely clear, since dose estimates-with either of the two methods-were largely within tolerable limits. Nonetheless, PET images obtained before RIT showed significantly different patterns of liver involvement between these two patients: the liver of patient F showed up to 13 single well-defined lesions (Fig. 5), while the liver of patient $G$ exhibited a diffuse metastatic spread, without clearly defined foci of FDG uptake (Fig. 6). Therefore, it may be possible that, due to the apparently more diffuse tumor infiltrate, the liver of patient $G$ was more compromised than the liver of patient F. Unfortunately, we did not have a direct comparison of liver toxicity between patients $F$ and G; we only could observe that, unlike patient $G$, patient $F$ conserved unremarkable liver function indices until death, which occurred 60 days after RIT-Z.

To our knowledge, there is no literature addressing different radiosensitivities for organs with tumor involvement. In fact, in EBRT, metastatic organs conserve the $\alpha / \beta$ parameters of healthy organs. In our opinion, this would be an interesting and almost unexplored field of research.

Finally, we would like to discuss some methodological differences between the present work and previous voxelbased dosimetric studies.

In the present article, 3D dose calculations were performed using the voxel $S$ value method. ${ }^{27}$ It is worth noting that other algorithms, such as the dose point kernel convolution $^{49,50}$ and the direct Monte Carlo radiation transport technique $^{27}$ are available and often implemented in clinical practice. However, the voxel $S$ value and the dose pointkernel algorithms are generally considered to be good compromises between simplified model-based calculations and more resource-intensive approaches based on Monte Carlo radiation transport. ${ }^{49}$ In addition, they are likely to provide similar results. ${ }^{27,49-51}$ Recently, a dose calculation algorithm that assumes that kinetic energy is deposited in the same voxel where particles are emitted has been described. ${ }^{52}$ Interestingly, this latter study demonstrates that dosimetry using explicit Monte Carlo calculations is not always warranted, since the limited spatial resolution of the SPECT imaging system is in the same order of magnitude as compared to the $\beta$ particle ranges. ${ }^{52}$

Our choice of a 9.3- $\mathrm{mm}^{3}$ voxel yielded a high self-dose within the source voxel, while $S$ values of adjacent voxels decrease rapidly. ${ }^{20}$ As a consequence, the convolution of a high $S$ value in the centroid voxel would produce approximately the same result than considering that all kinetic energy is deposited within the voxel where the decay has occurred (local absorption of dose).

We acknowledge that, with such voxel size, no conclusions about heterogeneous activity distributions below $0.8 \mathrm{~mL}$ (i.e., the voxel volume) can be drawn. The impact of a heterogeneous submillimetric dose distribution is hardly quantifiable and goes beyond the scope of the present study. However, as a general rule of thumb, the spatial scale of heterogeneity depends on the physical properties of radiation. If there are a large number of $\beta$ particle tracks (as is the case of tumor areas, which show regions of high uptake), it is likely that the constituents of individual tracks occupy overlapping physical volumes, therefore approaching a homogeneous distribution. ${ }^{53}$ This may be particularly true when the $\beta$ particle range is larger than-or approximately the same size as-the voxel dimension. This is the case of the present study, where the cross-fire deriving from ${ }^{90} \mathrm{Y}$ electrons (maximal range 
$\sim 11 \mathrm{~mm}$ ) is likely to reduce intra-voxel heterogeneities. Hence, in our opinion, though large, a 9.3-mm cubic voxel is not an obstacle for tumor contouring and is not likely to affect dose calculations since, to a first approximation, intravoxel heterogeneity may be assumed negligible.

As most recent guidelines ${ }^{28}$ recommend, we included PET/CT in the response evaluation to better account for tumor metabolism. Changes in glucose uptake are indeed more closely related to cell killing/regrowth rates than changes in tumor volume. The combined utilization of morphological and metabolic criteria for response assessment represents one of the major differences between the present study and the previous reports on Bexxar..$^{9-12,14,15}$ In the absence of a hybrid SPECT/CT scanner, we decided to contour tumors on SPECT images rather than on CT axial slices for activity quantification. Our decision was based on the assumption that tumor volumes calculated on SPECT images might better correspond to the actual tumor than noncontrast enhanced, low-dose CT images. Volumes derived by CT were used for partial-volume corrections and activity recovery, performed through the application of volume thresholds and activity RCs, respectively. After proper corrections, SPECTbased tumor volumes were in good agreement with those assessed from CT, with a maximum overestimation of $13 \%$ for the smallest lesion. Dedicated phantom studies, assessing the agreement between nominal and SPECT-evaluated volumes, were presented elsewhere. ${ }^{20}$

Also, differently from previous reports on RIT-B dosimetry, ${ }^{14,15}$ we did not consider the potential contribution of tumor shrinkage and unlabelled antibody during therapy on dose calculations. Although indolent NHLs are potentially sensitive to rituximab alone even after RIT-Z, ${ }^{47}$ tumor shrinkage during pretherapeutic imaging or soon after the administration of the therapeutic activity has not yet been recorded in RIT-Z. Interestingly, with RIT-B, more than 50\% volume reduction was observed after the administration of unlabelled tositumomab in previously untreated lesions, ${ }^{10}$ while in relapsed patients the contribution of cold tositumomab was less, but still significant. ${ }^{11,14,15}$ Systematic use of SPECT/CT in the pretherapeutic dosimetry of RIT-Z should be able to address the issue of any possible contribution of unlabelled rituximab.

\section{Acknowledgments}

Preliminary data were presented at the 10th Congress of the Italian Association of Nuclear Medicine (AIMN, Rimini 2011) and published in an abstract form, ref: Cicone $F$, D'Arienzo M, Carpaneto A, Papa A, Cottignoli C, Russo E, et al. Voxel-based dosimetry of ${ }^{90} \mathrm{Y}$-Ibritumomab-tiuxetan: application of the EUD concept to target lesions. Q J Nucl Med Mol Imaging 2011;55(Suppl 1): 14.

\section{Disclosure Statement}

There are no conflicts of interest to disclose.

\section{References}

1. Witzig TE, Gordon LI, Cabanillas F, et al. Randomized controlled trial of yttrium-90-labeled ibritumomab tiuxetan radioimmunotherapy versus rituximab immunotherapy for patients with relapsed or refractory low-grade, follicular, or transformed B-cell non-Hodgkin's lymphoma. J Clin Oncol 2002;20:2453.

2. Fisher RI, Kaminski MS, Wahl RL, et al. Tositumomab and iodine-131 tositumomab produces durable complete remissions in a subset of heavily pretreated patients with lowgrade and transformed non-Hodgkin's lymphomas. J Clin Oncol 2005;23:7565.

3. Wahl RL, Kroll S, Zasadny KR. Patient-specific whole-body dosimetry: Principles and a simplified method for clinical implementation. J Nucl Med 1998;39(8 Suppl):14S.

4. Wiseman GA, Kornmehl E, Leigh B, et al. Radiation dosimetry results and safety correlations from 90Y-ibritumomab tiuxetan radioimmunotherapy for relapsed or refractory nonHodgkin's lymphoma: Combined data from 4 clinical trials. J Nucl Med 2003;44:465.

5. Conti PS, White C, Pieslor P, et al. The role of imaging with (111)In-ibritumomab tiuxetan in the ibritumomab tiuxetan (zevalin) regimen: Results from a Zevalin Imaging Registry. J Nucl Med 2005;46:1812.

6. Cremonesi M, Ferrari M, Grana CM, et al. High-dose radioimmunotherapy with ${ }^{90} \mathrm{Y}$-ibritumomab tiuxetan: Comparative dosimetric study for tailored treatment. J Nucl Med 2007;48:1871.

7. Chiesa C, Botta F, Coliva A, et al. Absorbed dose and biologically effective dose in patients with high-risk nonHodgkin's lymphoma treated with high-activity myeloablative ${ }^{90}$ Y-ibritumomab tiuxetan (Zevalin). Eur J Nucl Med Mol Imaging 2009;36:1745.

8. Pacilio M, Betti M, Cicone F, et al. A theoretical doseescalation study based on biological effective dose in radioimmunotherapy with (90)Y-ibritumomab tiuxetan (Zevalin). Eur J Nucl Med Mol Imaging 2010;37:862.

9. Koral KF, Dewaraja Y, Li J, et al. Update on hybrid conjugate-view SPECT tumor dosimetry and response in 131I-tositumomab therapy of previously untreated lymphoma patients. J Nucl Med 2003;44:457.

10. Koral KF, Francis IR, Kroll S, et al. Volume reduction versus radiation dose for tumors in previously untreated lymphoma patients who received iodine-131 tositumomab therapy. Conjugate views compared with a hybrid method. Cancer 2002;94(4 Suppl):1258.

11. Sgouros G, Squeri S, Ballangrud AM, et al. Patient-specific, 3-dimensional dosimetry in non-Hodgkin's lymphoma patients treated with 131I-anti-B1 antibody: Assessment of tumor dose-response. J Nucl Med 2003;44:260.

12. Prideaux AR, Song H, Hobbs RF, et al. Three-dimensional radiobiologic dosimetry: Application of radiobiologic modeling to patient-specific 3-dimensional imaging-based internal dosimetry. J Nucl Med 2007;48:1008.

13. Niemierko A. Reporting and analyzing dose distributions: A concept of equivalent uniform dose. Med Phys 1997;24:103.

14. Amro H, Wilderman SJ, Dewaraja YK, et al. Methodology to incorporate biologically effective dose and equivalent uniform dose in patient-specific 3-dimensional dosimetry for non-Hodgkin lymphoma patients targeted with ${ }^{131} \mathrm{I}-$ tositumomab therapy. J Nucl Med 2010;51:654.

15. Dewaraja YK, Schipper MJ, Roberson PL, et al. 131Itositumomab radioimmunotherapy: Initial tumor doseresponse results using 3-dimensional dosimetry including radiobiologic modeling. J Nucl Med 2010;51:1155.

16. Wiseman GA, White CA, Stabin M, et al. Phase I/II ${ }^{90} \mathrm{Y}$ Zevalin (yttrium-90 ibritumomab tiuxetan, IDEC-Y2B8) radioimmunotherapy dosimetry results in relapsed or refractory non-Hodgkin's lymphoma. Eur J Nucl Med 2000;27:766. 
17. Knox SJ, Goris ML, Trisler K, et al. Yttrium-90-labeled antiCD20 monoclonal antibody therapy of recurrent B-cell lymphoma. Clin Cancer Res 1996;2:457.

18. Iagaru A, Gambhir SS, Goris ML. 90Y-ibritumomab therapy in refractory non-Hodgkin's lymphoma: Observations from 111In-ibritumomab pretreatment imaging. J Nucl Med 2008; 49:1809.

19. Delaloye AB, Antonescu C, Louton T, et al. Dosimetry of 90Y-ibritumomab tiuxetan as consolidation of first remission in advanced-stage follicular lymphoma: Results from the international phase 3 first-line indolent trial. J Nucl Med 2009;50:1837.

20. D'Arienzo M, Cicone F, Chiacchiararelli L, et al. Threedimensional patient specific dosimetry in radioimmunotherapy with 90Y-ibritumomanb-tiuxetan. Cancer Biother Radiopharm 2012;27:124.

21. Ogawa K, Harata Y, Ichihara T, et al. A practical method for position-dependent Compton scatter correction in single photon emission CT. IEEE Trans Med Imaging 1991;10:408.

22. Brown S, Bailey DL, Willowson $K$, et al. Investigation of the relationship between linear attenuation coefficients and CT Hounsfield units using radionuclides for SPECT. Appl Radiat Isot 2008;66:1206.

23. Mortelmans L, Nuyts J, Van Pamel G, et al. A new thresholding method for volume determination by SPECT. Eur J Nucl Med 1986;12:284.

24. Erdi YE, Wessels BW, Loew MH, et al. Threshold estimation in single photon emission computed tomography and planar imaging for clinical radioimmunotherapy. Cancer Res 1995;55(23 Suppl):5823s.

25. Krak NC, Boellaard R, Hoekstra OS, et al. Effects of ROI definition and reconstruction method on quantitative outcome and applicability in a response, monitoring trial. Eur J Nucl Med Mol Imaging 2005;32:294.

26. Koral KF, Dewaraja Y. I-131 SPECT activity recovery coefficients with implicit or triple-energy-window scatter correction. Nucl Instrum Methods Phys Res A 1999;422:688.

27. Bolch WE, Bouchet LG, Robertson JS, et al. MIRD pamphlet No. 17: The dosimetry of nonuniform activity distributionsradionuclide $S$ values at the voxel level. Medical Internal Radiation Dose Committee. J Nucl Med 1999;40:11S.

28. Juweid ME, Stroobants S, Hoekstra OS, et al. Use of positron emission tomography for response assessment of lymphoma: Consensus of the Imaging Subcommittee of International Harmonization Project in Lymphoma. J Clin Oncol 2007; 25:571.

29. Cheson BD, Pfistner B, Juweid ME, et al. Revised response criteria for malignant lymphoma. J Clin Oncol 2007;25:579.

30. Siegel JA, Thomas SR, Stubbs JB, et al. MIRD pamphlet no. 16: Techniques for quantitative radiopharmaceutical biodistribution data acquisition and analysis for use in human radiation dose estimates. J Nucl Med 1999;40:37S.

31. Bujis WC, Siegel JA, Boerman OC, et al. Absolute organ activity estimate by five different methods of background correction. J Nucl Med 1998;39:2167.

32. Stabin MG, Sparks RB, Crowe E. OLINDA/EXM: The second generation personal computer software for internal dose assessment in nuclear medicine. J Nucl Med 2005;46:1023.

33. O'Donoghue JA. Implications of nonuniform tumor doses for radioimmunotherapy. J Nucl Med 1999;40:1337.

34. Barone R, Borson-Chazot F, Valkema R, et al. Patient specific dosimetry in predicting renal toxicity with ${ }^{90}$ YDOTATOC: Relevance of kidney volume and dose rate in finding a doseeffect relationship. J Nucl Med 2005;46:99S.
35. Zaider M, Amols HI. Practical considerations in using calculated healthy-tissue complication probabilities for treatmentplan optimization. Int J Radiat Oncol Biol Phys 1999;44:439.

36. Nahum AE. Microdosimetry and radiocurability: Modelling targeted therapy with beta-emitters. Phys Med Biol 1996; 41:1957.

37. Ebert MA, Zavgorodni SF. Modeling dose response in the presence of spatial variations in dose rate. Med Phys. 2000;27:393-400.

38. Strigari L, D'Andrea M, Maini CL, et al. Biological optimization of heterogeneous dose distributions in systemic radiotherapy. Med Phys 2006;33:1857.

39. Kalogianni E, Flux GD, Malaroda A. The use of BED and EUD concepts in heterogeneous radioactivity distributions on a multicellular scale for targeted radionuclide therapy. Cancer Biother Radiopharm 2007;22:143.

40. Semenenko VA, Reitz B, Day E, et al. Evaluation of a commercial biologically based IMRT treatment planning system. Med Phys 2008;35:5851.

41. Cuocolo A. Current research in nuclear medicine and molecular imaging in Italy: Highlights of the 10th National Congress of the Italian Association of Nuclear Medicine and Molecular Imaging. Q J Nucl Med Mol Imaging 2011;55:280.

42. Pouget JP, Navarro-Teulon I, Bardiès M, et al. Clinical radioimmunotherapy-the role of radiobiology. Nat Rev Clin Oncol 2011;8:720.

43. Murthy V, Thomas K, Foo K, et al. Efficacy of palliative lowdose involved-field radiation therapy in advanced lymphoma: A phase II study. Clin Lymphoma Myeloma 2008;8:241.

44. Haas RL, Poortmans P, de Jong D, et al. High response rates and lasting remissions after low-dose involved field radiotherapy in indolent lymphomas. J Clin Oncol 2003;21:2474.

45. Morschhauser F, Illidge T, Huglo D, et al. Efficacy and safety of yttrium-90 ibritumomab tiuxetan in patients with relapsed or refractory diffuse large B-cell lymphoma not appropriate for autologous stem-cell transplantation. Blood 2007;110:54.

46. Cicone F, Baldini R, Cox MC, et al. Radioimmunotherapy of heavily pre-treated, non-Hodgkin's lymphoma patients: Efficacy and safety in a routine setting. Anticancer Res 2009; 29:4771.

47. Cicone F, Russo E, Carpaneto A, et al. Follicular Lymphoma at relapse after rituximab containing regimens: Comparison of time to event intervals prior to and after 90Y-IbritumomabTiuxetan. Hematol Oncol 2011;29:131.

48. Assié K, Dieudonné A, Gardin I, et al. Comparison between $2 \mathrm{D}$ and $3 \mathrm{D}$ dosimetry protocols in 90Y-ibritumomab tiuxetan radioimmunotherapy of patients with non-Hodgkin's lymphoma. Cancer Biother Radiopharm 2008;23:53.

49. Giap HB, Macey DJ, Bayouth JE, et al. Validation of a dosepoint kernel convolution technique for internal dosimetry. Phys Med Biol. 1995;40:365.

50. Prestwich WV, Nunes J, Kwok CS. Beta dose point kernels for radionuclides of potential use in radioimmunotherapy. J Nucl Med 1989;30:1036.

51. Dewaraja YK, Frey EC, Sgouros G et al. MIRD Pamphlet No. 23: Quantitative SPECT for Patient-Specific 3-Dimensional Dosimetry in Internal Radionuclide Therapy. J Nucl Med 2012;53:1310.

52. Ljungberg M, Sjögreen-Gleisner K. The accuracy of absorbed dose estimates in tumours determined by quantitative SPECT: A Monte Carlo study. Acta Oncol 2011;50:981.

53. Heterogeneity: Physical, chemical, and biological consideration. J ICRU 2011;11:17. 